\title{
A Ventricular Far-Field Artefact Filtering Technique for Atrial Electrograms
}

\author{
Simanto Saha ${ }^{1,2}$, Simon Hartmann ${ }^{1}$, Dominik Linz ${ }^{1,2}$, Prashanthan Sanders ${ }^{1,2}$, Mathias Baumert ${ }^{1}$ \\ ${ }^{1}$ The University of Adelaide, Adelaide, Australia \\ ${ }^{2}$ Royal Adelaide Hospital, Adelaide, Australia
}

\begin{abstract}
Intracardiac atrial electrograms (EGM) are prone to ventricular far-field potentials due to ventricular depolarization. In this study, a filtering technique integrating independent component analysis (ICA) and wavelet decomposition has been proposed to significantly reduce the ventricular far-field contents while preserving the EGM morphology related to atrial activations. First, the wavelet decomposition is applied to each unipolar EGM. Then, ICA is applied to the decomposed unipolar EGM components and surface ECG template. Each independent component is cross-correlated with the simultaneously recorded ECG template and the three components with higher correlation coefficients were eliminated before applying inverse ICA. Total of 126 unipolar EGM collected from an atrial fibrillation patient have been included. Results indicate that the proposed filtering can reduce the ventricular signal power by around $17 d B$ (decibel). Furthermore, the signal-tonoise ratio is increased by approximately $17 \mathrm{~dB}$ after applying the proposed filtering. In conclusion, the proposed filtering method could be used for atrial fibrillation-related intracardiac mapping for catheter ablation. Further studies on a larger dataset are essential to quantify the exact impact of ventricular artefacts on both unipolar and bipolar EGM and the effectiveness of the proposed filtering technique.
\end{abstract}

\section{Introduction}

Atrial fibrillation (AF) is the most occurring heart rhythm disorder in humans [1]. Remodeling of the arrhythmogenic substrates that potentially interrupt the normal cardiac electrical wave propagation dynamics during sinus rhythm is one of the primary causes of AF induction and prolongation [2]. Intracardiac catheter ablation is a widely used clinical procedure to terminate AF-related atrial electrical sources and to reinstate sinus rhythm. As a precursor to any catheter ablation procedure, intracardiac electrograms (EGM) are recorded using catheter-based electrodes to determine AF-related sources as target ablation sites.

Ventricular far-field potentials often influence local
EGM recordings. Specifically, unipolar EGM are more likely to be influenced by the ventricular artefacts. Although current mapping systems use bipolar EGM that are assumed to be less sensitive to the far-field potentials, bipolar EGM are constructed from unipolar EGM. Thus, it is important to characterize the influence of inevitable ventricular far-field effects on local unipolar EGM. It is essential to further evaluate the effects on bipolar EGM.

Rieta and Hornero have studied the effect of ventricular activities on both unipolar and bipolar EGM signal and described three different techniques for removing ventricular artefacts [3]. The techniques are independent component analysis (ICA)-based filtering, template matching, and subtraction and adaptive ventricular cancellation.

In this study, a combination of ICA and wavelet decomposition (WD) has been utilized to reduce the effects of ventricular contents on local unipolar EGM. Previous studies integrated wavelet transform and independent component analysis for source separation in single channel recording $[4,5]$. The underlying motivation of using ICA is that atrial and ventricular activities can be considered statistically independent originated from two distinct sources [6]. In that case, in the electrode output, i.e., unipolar EGM is the mixture of both atrial and ventricular contents.

\section{Methods}

\subsection{Intracardiac electrogram acquisition}

This study had been approved by the ethics committee of the Royal Adelaide Hospital. An array of 18 unipolar electrodes (EnSite ${ }^{\mathrm{TM}}$ HD Grid Catheter, Abbott Medical) were used to record the left atrial intracardiac EGM from a patient undergoing catheter ablation due to symptomatic drug-refractory persistent AF. Figure 1 shows the configuration of the electrode array. The electrodes are oriented in four parallel struts in such a way to maintain the centreto-centre inter-electrode spacing of $4 \mathrm{~mm}( \pm 0.5 \mathrm{~mm})$ between any two adjacent electrodes along and across the strut. The length of each electrode is $1 \mathrm{~mm}$ with an outer diameter of $0.81 \mathrm{~mm}$. The sampling rate was set at 2034.5 $\mathrm{Hz}$. 


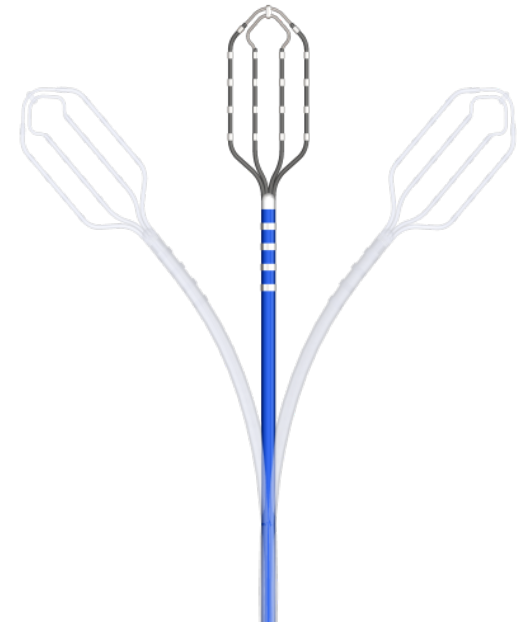

Figure 1. An array of 18 unipolar electrodes used to collect the intracardiac EGM.

The EGM were acquired for 3 seconds for each shot along with surface ECG. Each shot consists of simultaneously recorded 18 unipolar EGM. A total of 7 shots were included in this analysis after visually inspecting them for the annotation of significant contamination of ventricular far-field potentials in local atrial EGM. As a result, the performance of the proposed filtering technique has been validated on 126 unipolar recordings.

\subsection{Ventricular artefact reduction method}

Figure 2 illustrates the basic block diagram of the proposed filtering method for ventricular artefact reduction. The raw unipolar EGM were first filtered using a Bandpass (Butterworth) filter of order 4 . The corner frequencies were $5 \mathrm{~Hz}$ and $1 \mathrm{kHz}$. The low cut-off was used to eliminate any baseline drift from the raw EGM and the high cutoff is selected to maintain the Nyquist rate (approximately half of the sampling rate). Then, each unipolar EGM was decomposed up to level 6 using wavelet transform. The used wavelet basis was Coiflet 5 as its morphological resemblance to QRS complex morphology. Simultaneously recorded lead II ECG $(30-300 \mathrm{~Hz})$ was considered as a template for characterizing ventricular contents, i.e., QRS event related to ventricular depolarization. The lead II was selected because it provides a relatively higher amplitude as compared to other ECG leads. A notch filter was applied to eliminate powerline interference from both EGM and ECG.

ICA was then applied to the 8 signal contents including the 7 contents of unipolar EGM extracted by wavelet decomposition and one simultaneously recorded ECG. As a blind source separation technique, ICA works with $N$ ob-

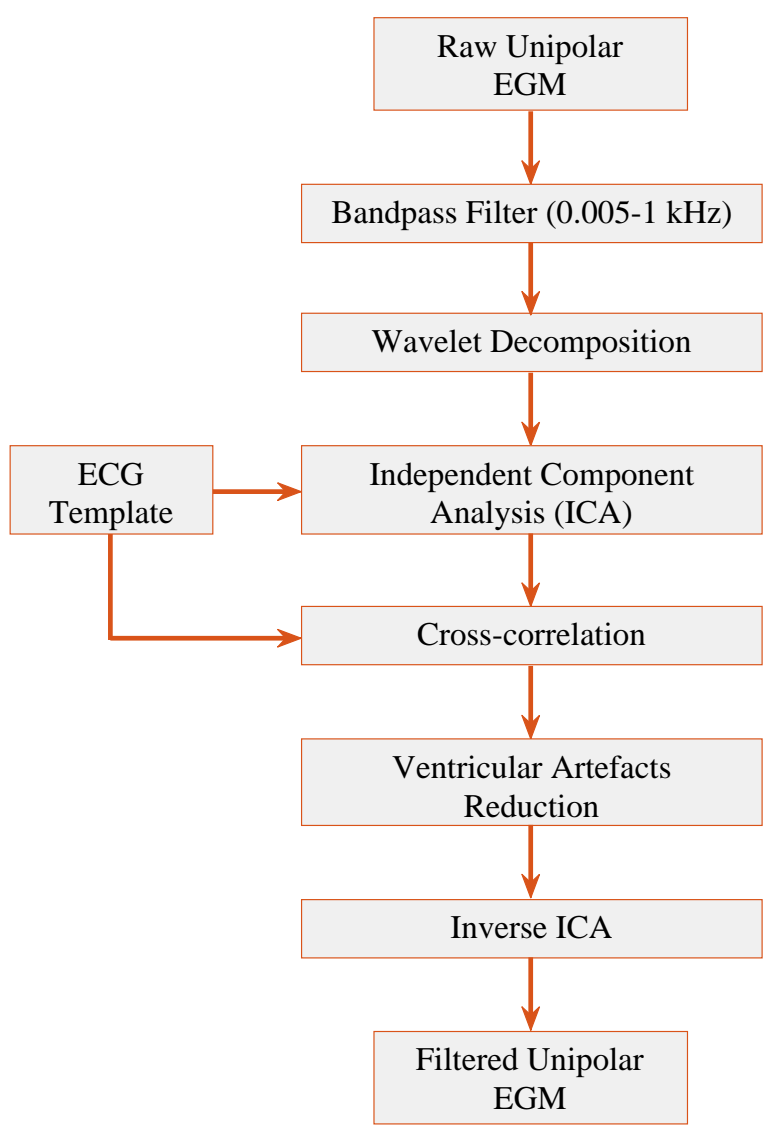

Figure 2. A basic block diagram illustrating the proposed filtering technique integrating independent component analysis and wavelet decomposition.

served signals that are considered as mixtures of multiple signal contents from diverse independent sources. Assuming $x(t) \in \mathbb{R}^{\mathbb{M}}$ represents the observed signals in $M$ sensors (i.e., electrodes) and $s(t) \in \mathbb{R}^{\mathbb{N}}$ represents $N$ source signals, then the linear mixtures model for ICA decomposition can be written as follows [7]

$$
x(t)=A s(t) .
$$

Here, $A \in \mathbb{R}^{\mathbb{M} \times \mathbb{N}}$ is the unknown mixing matrix that has to be estimated. Different ICA algorithms work by assuming the statistical independence of the source signals. In this study, the FastICA algorithm is used. FastICA transforms the observed signals by maximizing non-gaussianity of the components, which is an alternative manifestation of statistical independence. For more details of FastICA algorithm, please refer to $[7,8]$.

Each of the eight independent components was crosscorrelated with the ECG template. Finally, three independent components showing higher cross-correlation were eliminated proceeding to inverse ICA to reconstruct the filtered EGM. 

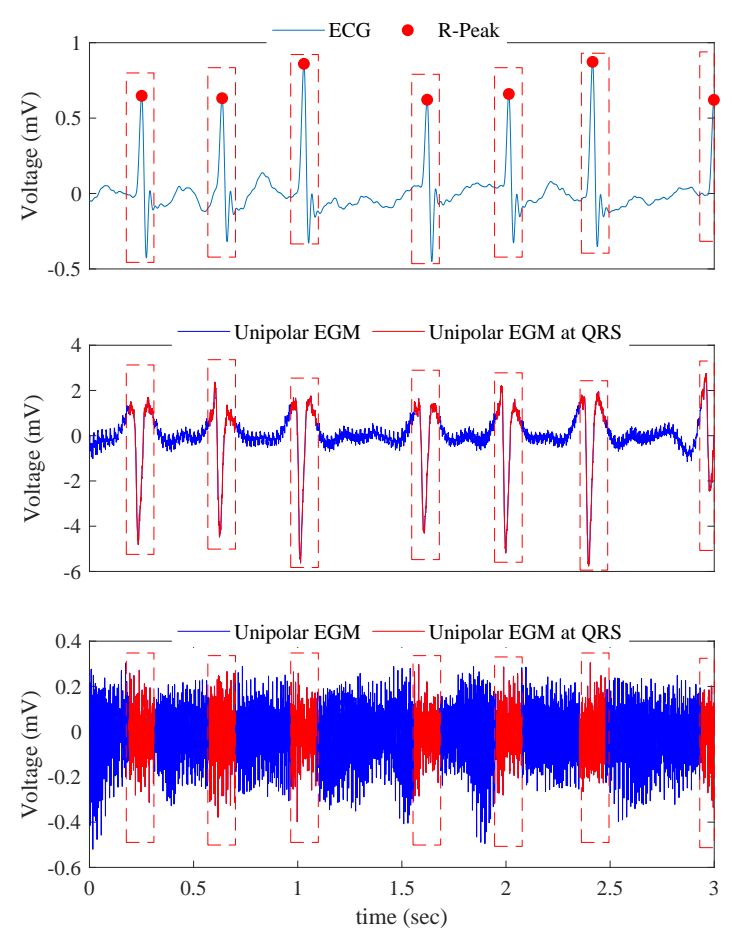

Figure 3. Illustration of defining ventricular signal contents as characterized by QRS complexes, top-bottom: lead II surface ECG and R-peak annotations, signal and noise contents before and after ventricular artefact elimination (Note: a $125 \mathrm{~ms}$ window is placed at each QRS event to define the ventricular signal).

\subsection{Performance evaluation}

The performance of the proposed filtering technique was evaluated by estimating the reduction in signal power in QRS events as defined by a $125 \mathrm{~ms}$ window. Figure 3 depicts the definition of the ventricular contents (characterized by QRS complex) and the contents of the remaining signal. Ventricular artefact power was calculated on signal contents in the QRS-defined windows. Furthermore, the signal-to-noise ratio (SNR) was calculated by considering the signal contents at QRS events as noise and the remaining signal contents as a signal. It is to be noted that the SNR measurement would be useful to investigate the contribution of ventricular far-field potentials in local atrial EGM.

Finally, statistical analysis was carried out to evaluate if the power at QRS-defined signal contents and SNR were significantly different. The Lilliefors test was applied to check the distributions of the power and SNR values with and without filtering. As the distributions do not follow Gaussianity, the Mann-Whitney U test was applied. Pvalues $<0.01$ were considered significant.

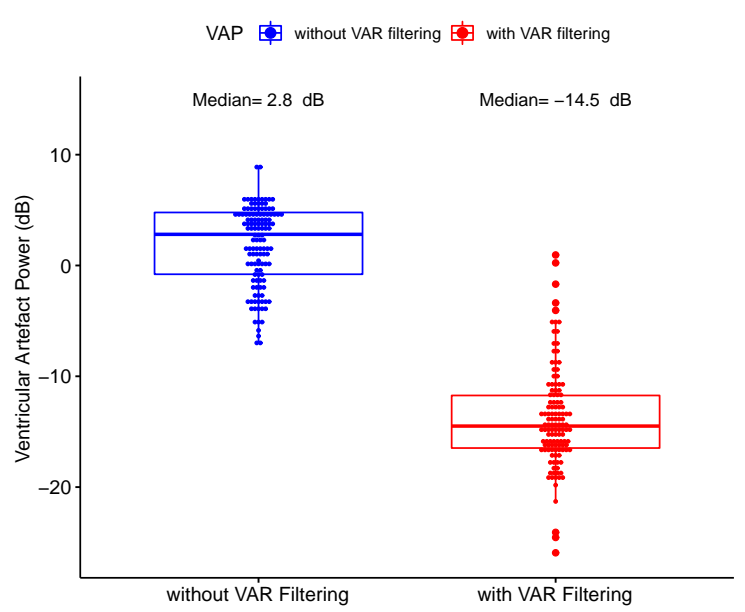

Figure 4. Comparison of the power of ventricular contents before and after ventricular artefact reduction filtering (VAR: ventricular artefact reduction).

\section{Results and discussion}

Figure 3 delineates the effectiveness of the proposed filtering technique for ventricular artefact reduction. Specifically, the significant reduction of signal amplitudes in the QRS-defined ventricular artefacts implicates the potential use of the proposed method during intracardiac mapping for AF catheter ablation. The unipolar EGM included in this study are significantly contaminated with ventricular artefacts, i.e., QRS-defined events due to ventricular depolarization.

Figure 4 compares the power of QRS-defined ventricular artefacts for all 126 unipolar EGM with and without applying the proposed filtering technique. Overall, the ventricular power is decreased by around $17 \mathrm{~dB}$ after applying the filtering. Figure 5 indicates an increase in SNR by approximately $17 \mathrm{~dB}$. Both the increase in SNR and the decrease in QRS-defined ventricular signal power evince the applicability of the proposed method in intracardiac mapping.

To date, there is no automatic filtering technique in commercially available mapping tools. Generally, the ventricular artefact reduction is done by visually inspecting the EGM in an electrophysiology laboratory, which is a tedious process. With the advancement of high density and high-resolution mapping tools, the proposition of an automated filtering method for ventricular artefact reduction would be practical. Typically, a band of $30-300 \mathrm{~Hz}$ is used clinically to extract meaningful signal attributes that are used to identify ablation targets. However, the clinical significance of the signal contents in the eliminated bands would require to be further studied for scrutinizing the effects of filtering in substrate-based AF ablation. As the 


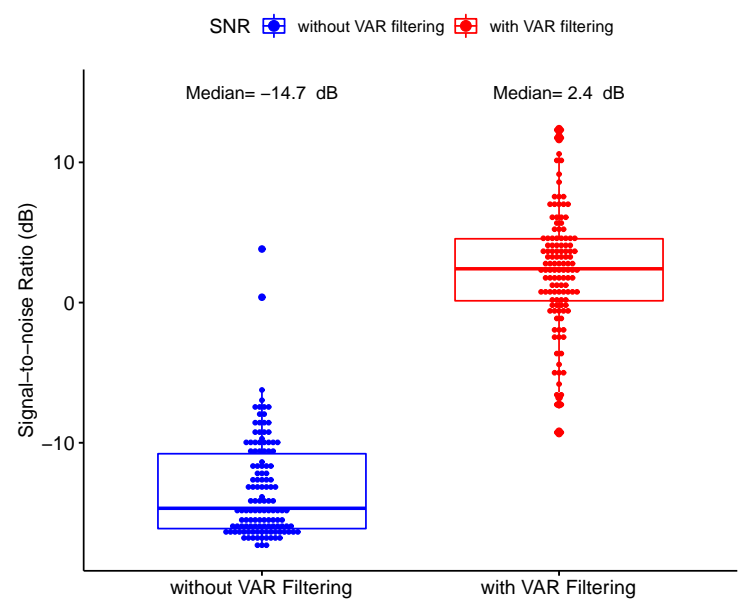

Figure 5. Comparison of the signal-to-noise ratio before and after ventricular artefact reduction filtering (VAR: ventricular artefact reduction).

peak-to-peak voltage is typically used for AF-related atrial substrate characterization, it might be critical to filter the raw unipolar EGM carefully while preserving local amplitude and morphology.

Bipolar EGM are considered less sensitive to ventricular far-field potentials relative to unipolar EGM and current cardiac mapping tools use bipolar EGM for atrial substrate characterization. There are multiple variables, including bipolar vector, inter-electrode spacing, electrode size and tissue contact that influence the interpretation of bipolar EGM $[9,10]$. Tissue contact depends on the accuracy of electrodes placement during signal acquisition although it is very difficult to maintain stable electrode placement in the presence of continuous blood flow and contraction of the heart muscles. As the bipolar EGM are constructed using unipolar EGM, it might be critical to carefully filter out undesired artefacts, i.e., ventricular far-field potentials while preserving the local EGM morphology for more accurate interpretation of bipolar EGM.

The elimination of three independent components was an arbitrary selection based on the experimental context. As mentioned earlier, all the unipolar EGM used in this study are severely contaminated with ventricular far-field effects. Notably, the unipolar EGM included in this preliminary study were selected from the atrial sites near the Mitral valve. Future studies may consider algorithms for adaptive elimination of independent components for better preserving the local EGM morphology.

\section{Conclusion}

Intracardiac unipolar EGM are usually contaminated with ventricular far-field effects that may cause misinter- pretation of AF-related target sources during catheter ablation. The proposed ICA and wavelet decomposition-based ventricular artefact reduction technique seems effective, which could be incorporated in the future mapping tools after further validation on a larger dataset.

\section{Acknowledgments}

Authors would like to thank the team of Abbott Medical for assisting raw data extraction.

\section{References}

[1] Baumert M, Sanders P, Ganesan A. Quantitativeelectrogram-based methods for guiding catheter ablation in atrial fibrillation. Proceedings of the IEEE 2016; 104(2):416-431.

[2] Lau DH, Linz D, Schotten U, Mahajan R, Sanders P, Kalman JM. Pathophysiology of paroxysmal and persistent atrial fibrillation: rotors, foci and fibrosis. Heart Lung and Circulation 2017;26(9):887-893.

[3] Rieta JJ, Hornero F. Comparative study of methods for ventricular activity cancellation in atrial electrograms of atrial fibrillation. Physiological measurement 2007;28(8):925.

[4] Mijovic B, De Vos M, Gligorijevic I, Taelman J, Van Huffel S. Source separation from single-channel recordings by combining empirical-mode decomposition and independent component analysis. IEEE transactions on biomedical engineering 2010;57(9):2188-2196.

[5] Hartmann S, Baumert M. Automatic a-phase detection of cyclic alternating patterns in sleep using dynamic temporal information. IEEE Transactions on Neural Systems and Rehabilitation Engineering Sep. 2019;27(9):1695-1703.

[6] Rieta JJ, Castells F, Sánchez C, Zarzoso V, Millet J. Atrial activity extraction for atrial fibrillation analysis using blind source separation. IEEE Transactions on Biomedical Engineering 2004;51(7):1176-1186.

[7] Hyvärinen A, Oja E. Independent component analysis: algorithms and applications. Neural networks 2000;13(45):411-430

[8] Hyvärinen A, Oja E. A fast fixed-point algorithm for independent component analysis. Neural computation 1997; 9(7):1483-1492.

[9] Anter E, Josephson ME. Bipolar voltage amplitude: what does it really mean? Heart Rhythm 2016;13(1):326-327.

[10] Saha S, Linz D, Sanders P, Baumert M. Beamforminginspired spatial filtering technique for intracardiac electrograms. In 2019 41st Annual International Conference of the IEEE Engineering in Medicine and Biology Society (EMBC). IEEE, 2019; .

Address for correspondence:

Simanto Saha

School of Electrical and Electronic Engineering,

The University of Adelaide, SA 5005, Australia

simanto.saha@adelaide.edu.au 